\title{
Patient Preparation, Vascular Access, and Guiding Catheter Selection
}

\author{
Fuminobu Yoshimachi and Yuji Ikari
}

\subsection{Patient Preparation}

Before cardiac catheterization, patient preparation and checking of general condition and appropriateness for procedure should be done. This may not always be possible in emergency cases however.

\subsubsection{Changing Clothes}

For elective procedure, nurses or assistants help a patient change clothes into hospital gown. However, for emergency cases, patients will usually be taken directly to the catheter laboratory in their usual attire.

\subsubsection{Medication}

Ideally, it should be confirmed that daily oral medicine is taken by the patient correctly with particular attention to allergies.

\subsubsection{Dual Antiplatelet Therapy}

The use of dual antiplatelet therapy (DAPT) including aspirin and P2Y12 inhibitor must be checked. In case of emergency PCI, aspirin and prasugrel or ticagrelor should be given immediately.

\footnotetext{
F. Yoshimachi $\cdot$ Y. Ikari $(\bowtie)$

Department of Cardiology, Tokai University School of Medicine, Isehara, Japan

e-mail: ikari@is.icc.u-tokai.ac.jp
} 


\subsubsection{Metformin}

When daily oral medication includes metformin, this should be stopped $48 \mathrm{~h}$ after catheterization since it may cause lactic acidosis together with contrast medium. It is a well-known serious complication of contrast medium.

\subsubsection{Contrast Medium Allergy}

Although an idiosyncratic reaction, prior contrast allergy is important to note, and pre-procedural use of corticosteroids or antihistamine drugs should be considered if this has occurred previously. The presence of asthma does not predict risk of bronchospasm with contrast and does not need routine prophylaxis.

\subsubsection{Chronic Kidney Disease}

The association of contrast to contrast-induced nephropathy (CIN) and risk factors for this phenomenon are well documented; however, in the emergency setting, knowledge of background renal function is not always available. Intravenous fluids are an important strategy to reduce risk of CIN, but this may be contraindicated in some patients with STEMI or severe cardiac dysfunction. The maximal advisable dose of contrast medium is considered according to kidney function. Traditionally, body weight $(\mathrm{kg}) \times 5 /$ serum creatinine $(\mathrm{mg} / \mathrm{dL})$ is a maximum dose of contrast medium. Lower volumes of contrast reduce the likelihood of CIN.

\subsubsection{Anemia}

Anemia may be a sign of concealed gastrointestinal carcinoma. Since DAPT is required following PCI, gastrointestinal disease should be checked before elective PCI. This is not feasible in the emergency setting, although should blood results be available, the operator may wish to consider a stent where abbreviated DAPT is permissible or use a balloon angioplasty strategy.

\subsubsection{Blood Pressure/Oxygen Saturation Monitor/Venous Route}

Venous route is necessary for cardiac catheterization, but it should be selected not to disturb the approach site of PCI. Although one route is enough for most of the cases, two or three routes may be necessary for separate administration of continuous catecholamine and others. Saline or Ringer's solution is given as drip infusion. Be careful of volume overload in case of heart failure as less than $100 \mathrm{~mL} / \mathrm{h}$ is recommended for these cases. 
Although arterial pressure monitoring is possible, it is suggested to put manchette on arms or legs. In continuous monitoring of oxygen saturation, 12-lead electronic cardiogram should be put on.

\subsubsection{Emergency Cases}

Requisite minimum dose of oxygen should be given according to oxygen saturation and/or blood gas analysis [1]. For marked respiratory dysfunction, usually due to acute pulmonary edema which is refractory to immediate intravenous diuretics, intubation and ventilation may be required to facilitate the PCI procedure. Pressor support is sometimes required - usually where the patients have established or early cardiogenic shock. These patients require early comanagement with a cardiac intensivist to ensure that hemodynamic stability is maintained and optimized.

Emergency cases should be connected to a defibrillator - preferably using adhesive pads to allow rapid defibrillation during the PPCI procedure should VT or VF occur. This is particularly common at the reperfusion phase.

\subsection{Vascular Access}

\subsubsection{Access site}

Choice of access site is radial, femoral, or brachial arteries. Radial access is favorable because lower access site bleeding complications result in lower mortality rate in patients with acute coronary syndrome $[2,3]$. The second choice is femoral artery when radial access is not possible. Brachial access should be avoided because median nerve injury is a critical complication.

\subsubsection{Sheath Size}

A larger catheter caliber accepts bigger size devices or facilitates complex procedure such as simultaneous two-stent inflation. This has to be counterbalanced with increased chance of vascular complications - particularly bleeding. Recent studies have shown that bleeding complications have predict mortality rates; therefore slender size sheath is suggested for optimal safety. We generally recommend $6 \mathrm{~F}$ sheath and guide catheter which permit almost all types of complex PCI to be undertaken.

\subsubsection{Femoral Artery Puncture}

Puncture level proximal to the femoral bifurcation is suggested. However, the level of femoral bifurcation is widely varied in each patient. Femoral puncture 
point should be carefully determined. One technique is angio-guide positioning using the caput femoris. The other is ultrasound-guided positioning. Front wall puncture must be performed. Penetration to the back wall induces serious bleeding complication and is difficult to manage. It is important to ensure the guidewire correctly passes through the iliac artery and descending aorta under fluoroscopy because the external iliac artery takes a retroperitoneal course. Rarely the guidewire may cause branch perforation and serious bleeding complications, and therefore resistance to wire advancement needs careful and methodical evaluation.

Where there is femoral/iliac calcification, it is desirable to use a long sheath rather than a short sheath to facilitate catheter insertion and exchange and removal. Sometimes a very long sheath or a metallic coil or spring-type long sheath is required. However, we advise these be aspirated and flushed frequently as in situ thrombosis can occur, and this may be transported to the coronary circulation during catheter exchange.

\subsubsection{Radial Artery Puncture}

Radial artery puncture is often erroneously perceived to be difficult because it is smaller than femoral artery. Radial artery spasm and occlusion are not uncommon complications but can be managed effectively with meticulous attention to care. Notably though, radial artery access is safe because of straight anatomy, no significant branches, or very rare vascular complications such as bleeding - the vessel is easy to tamponade due to its superficial course and bony support posteriorly. Due to the lower complication rate, radial artery is considered as favorable approach site, particularly for primary PCI.

The puncture level is suggested as $1 \mathrm{~cm}$ above the radial process, but it is no problem to puncture above or below this point. Extremely low puncture may make application of hemostasis devices such as TR Band more challenging.

The use of sufficient local anesthetic (usually $1 \%$ lidocaine) may prevent radial artery spasm, by allowing arterial cannulation without pain. However, large bolus of anesthetic may make palpation of the artery more challenging. We generally use $1-1.5 \mathrm{~mL}$ of sc $1 \%$ lidocaine. Once the Seldinger wire is passed into the artery, additional sc lidocaine is delivered.

To overcome difficulty due to small caliber, the following techniques are considered to improve puncture efficiency:

\subsubsection{Ultrasound-Guided Radial Puncture}

Due to small caliber, radial artery spasm, or small size, radial puncture is sometimes challenging. Ultrasound-guided radial artery puncture is suggested with high probability of successful puncture [4] (Fig. 7.1). Checking the axis view of radial artery, the needle is advanced in the direction to push and crush the vessel. Even when spasm occurs or where radial palpation is not possible, the radial artery can almost always be detected and successfully punctured by ultrasound. 
Fig. 7.1 Echo-guided radial artery puncture

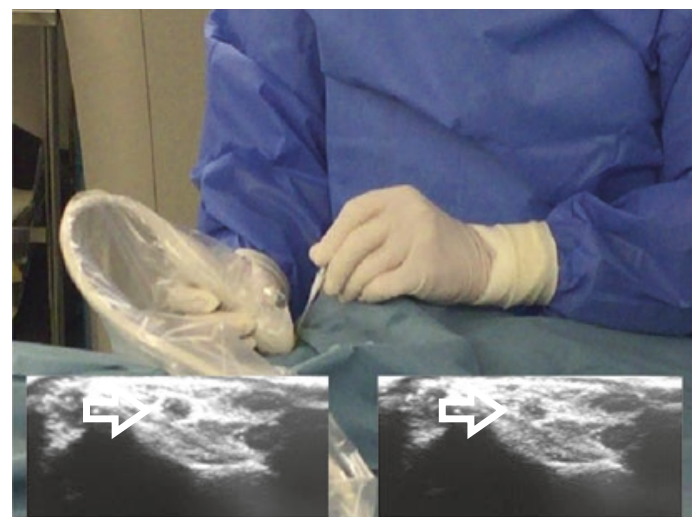

Fig. 7.2 Infrared light-assisted device called Mill Sus

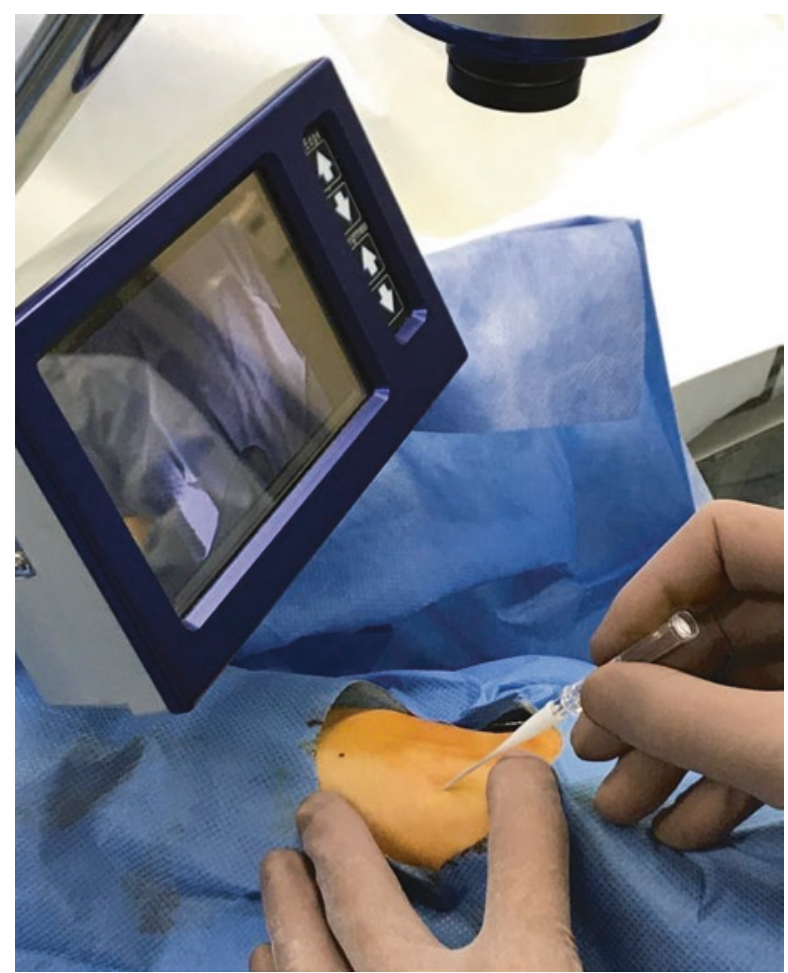

\subsubsection{Infrared Light-guided Puncture}

A recent technology has been developed to visualize radial artery using infrared light, named Mill Sus (Fig. 7.2). A purpose-designed camera detects transmitted near-infrared rays from the back of the wrist. The cardiologist can then puncture the visualized radial artery on the monitor. 


\subsubsection{Radial Artery Puncture in Cardiopulmonary Arrest}

It is very difficult to perform successful radial puncture in pulseless radial arteries due to shock or cardiopulmonary arrest.

\subsubsection{Angiography-guided Radial Artery Puncture}

In case of cardiopulmonary arrest (CPA), femoral artery puncture is also required for intra-aortic balloon pump (IABP) or extracorporeal membrane oxygenation (ECMO) insertion. After femoral access has been achieved and before inserting the IABP, a diagnostic catheter is inserted from the femoral into the subclavian artery to perform angiography of the upper limb. Because of CPA, contrast stasis in the arm allows fluoroscopic puncture of the radial artery.

\subsubsection{Kenzan Technique}

"Kenzan" is a classical, effective, and simple technique (Fig. 7.3).

Because of pulseless artery, the first puncture is made blindly. If first puncture is failed, with no backflow from needle, the needle should be left in situ. A second needle is then advanced next to the first needle. If no backflow is observed, a third needle is advanced next to the second needle. As many needles are used until backflow is observed. A mountain of needles appear like the Kenzan tool designed for Japanese flower arrangements to stand flowers. Quite aptly we call this technique "Kenzan."

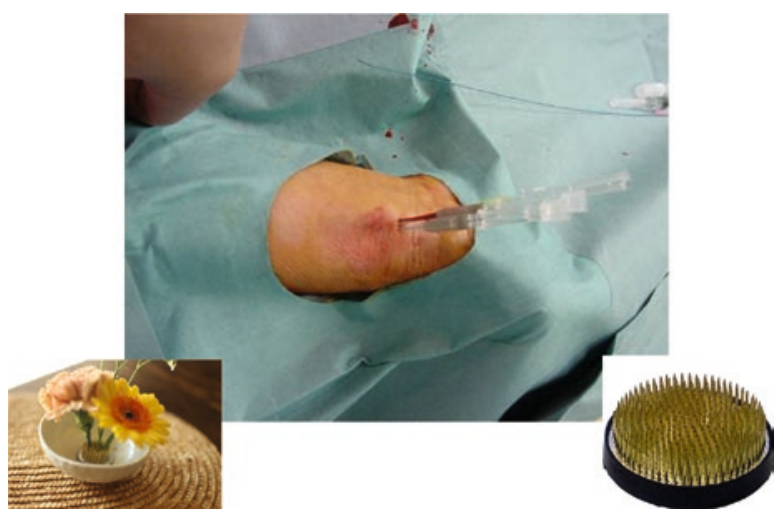

Fig. 7.3 Kenzan radial puncture technique. It is difficult to puncture pulseless radial artery in patients with cardiopulmonary arrest. In that case, puncture using several needles to the area of radial artery. One of the needles may be inside the radial artery. A kenzan, also called spiky frog, is a specific device used in the Japanese art of flower arrangement ikebana for fixing the flowers in the container. It consists of a heavy lead plate with erected brass needles where the stipes are fixed 
Fig. 7.4 Left distal transradial artery puncture. This is also called as proximal palmar arch puncture (PPAP)

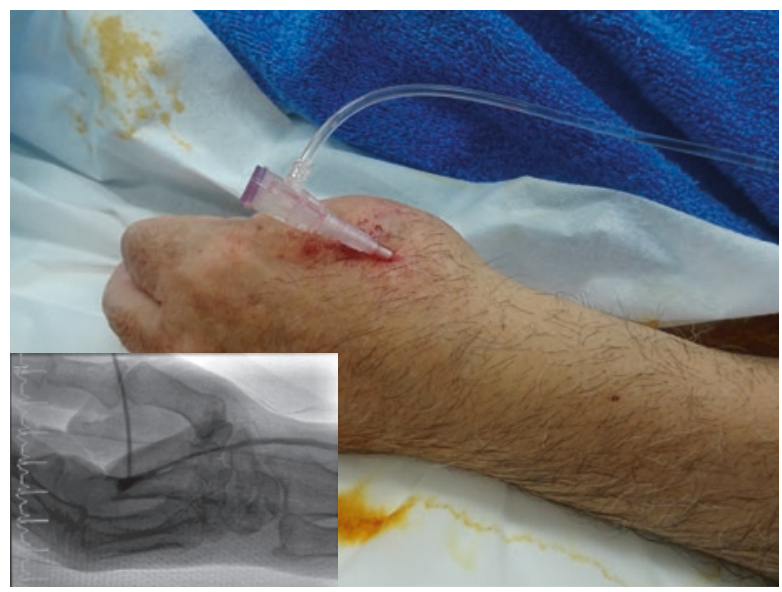

\subsubsection{Distal Radial Artery Puncture/Proximal Palm Artery Puncture}

This new method of approach has been introduced since 2016. Interchangeable terms of distal radial approach "d-TRI" or proximal palm artery puncture "PPAPp" are used [5] (Fig. 7.4). Hemostasis may be easy, and patient seems to have no discomfort after PCI. Long-term safety, feasibility, complication, and contraindications are unknown.

\subsection{Guiding Catheter Selection}

An appropriately selected guiding catheter is important for successful PCI either in transfemoral intervention (TFI) or transradial intervention (TRI). The radial approach presents a new set of challenges for the guiding catheter. It is important to understand the basic principles behind guiding catheter selection and the requirements for backup support, coaxial engagement, and the resulting procedural success that can be achieved when using a catheter specifically designed for access from the radial approach.

\subsection{Left Coronary Artery}

Performance of a guiding catheter can be assessed based on three factors: (1) easy and rapid engagement without specific manipulation, (2) strong backup force, and (3) safety not causing complications such as coronary or aortic dissection. An ideal guiding catheter should be easy to manipulate, supportive, and safe. 


\subsubsection{Judkins Left}

The Judkins left (JL) catheter is an excellent catheter because of its easy engagement and high safety margin. However, the backup force of JL in TRI is poor, although it fairs moderately better in TFI. Why is backup force of the JL in TRI so weak? One study on the physics of backup force in a guide catheter can answer this question (Fig. 7.5) [6]. This showed that the angle between the catheter and the reverse side of the aorta is a key factor to determine the backup force. The JL loses its angle-generating backup force when utilized in TRI. Thus, aside from simple interventions, this catheter is far from ideal in most TRI.

\subsubsection{Ikari Left}

The Ikari left (IL) guiding catheter was invented in 1995, first applied to PCI in 1996, and commercially available in 2002 [7]. The IL has three modifications from
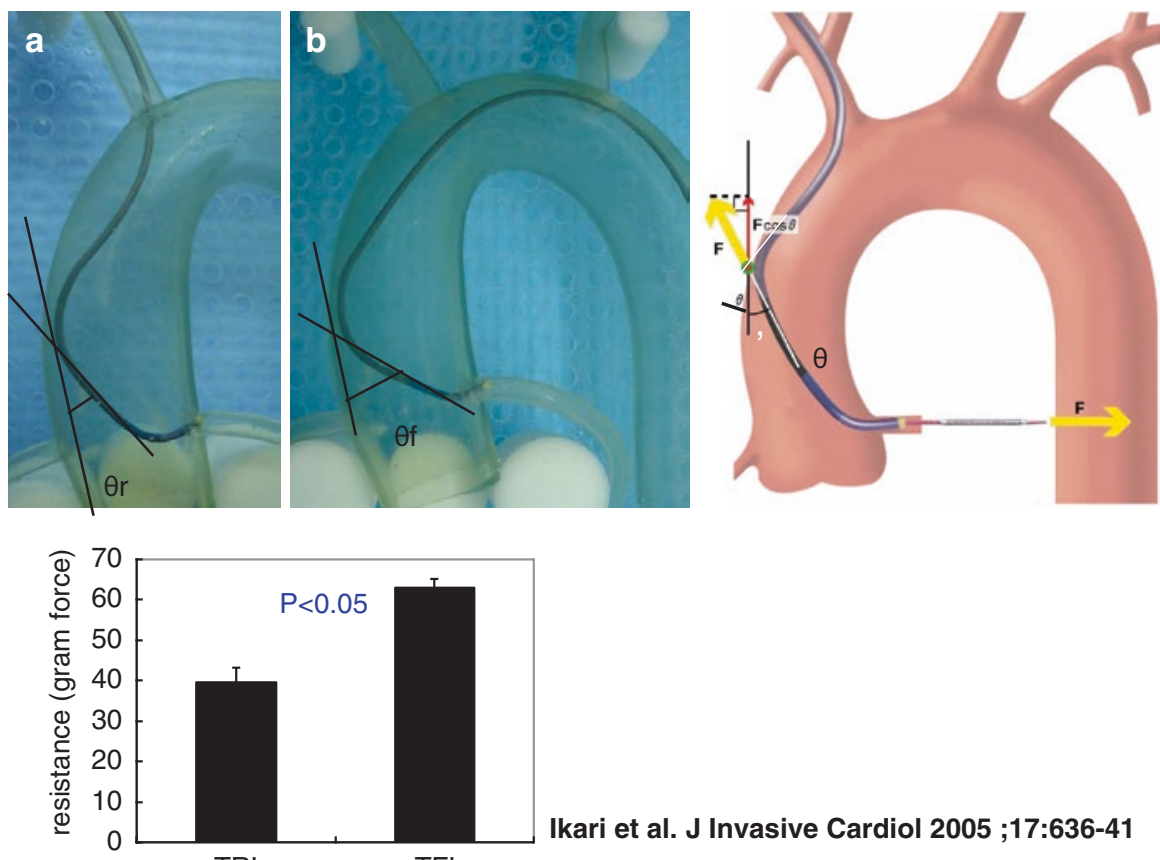

Ikari et al. J Invasive Cardiol 2005 ;17:636-41

TRI

TFI

Fig. 7.5 (a) Judkins left from right transradial approach. (b) Judkins left from femoral approach. (c) Force $(\mathrm{F})$ is necessary to pass a stent through a tight lesion. The force comes on the contralateral side of the aorta from where the catheter engages. The vertical segment of $\mathrm{F}$ is $\mathrm{F} \cos \theta \mathrm{T} \eta \varepsilon \varpi \varepsilon \rho \tau 1 \chi \alpha \lambda$

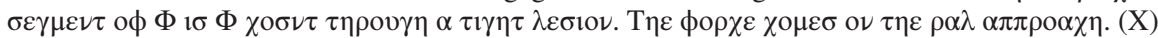

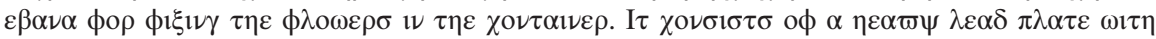
$\varepsilon \rho \varepsilon \chi \tau \varepsilon \delta \beta \rho(\mathbf{d})$. In vitro measurement of backup force of TRI and TFI using Judkins L 

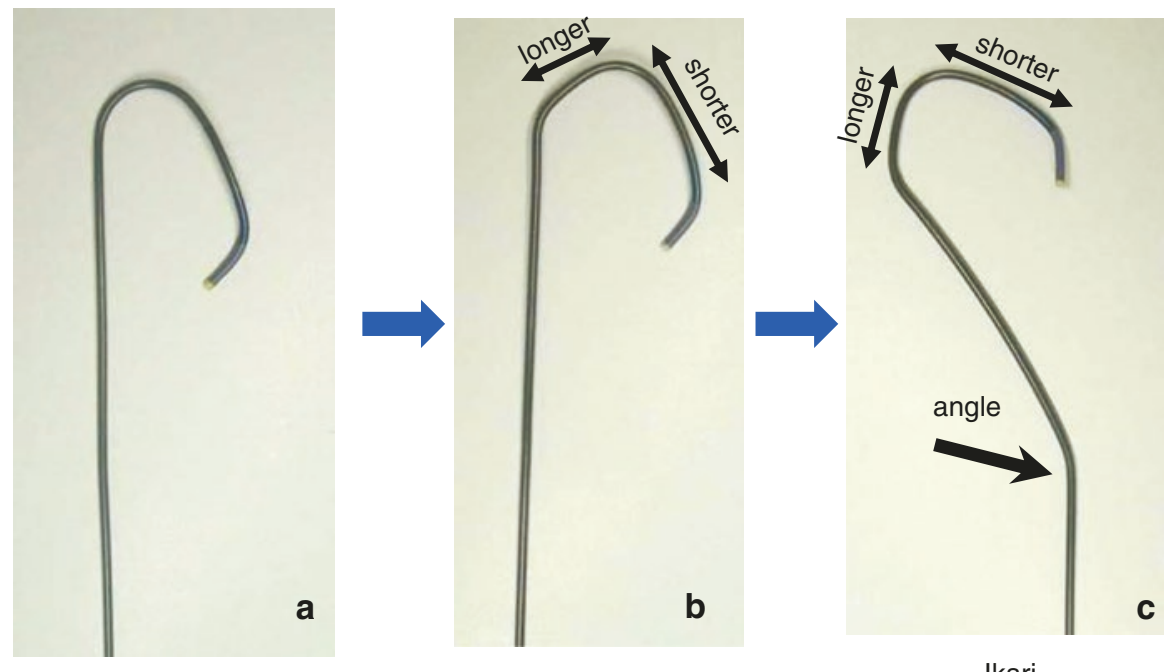

Judkins

Ikari

Fig. 7.6 Ikari left has three modifications compared with Judkins left

the JL: (1) shorter length between the third and the forth angles, (2) longer length between the second and the first angles, and (3) a new primary curve that was added to fit the brachiocephalic artery (Fig. 7.6). This means IL is a modified JR catheter designed specifically for TRI. Backup force of the IL is greater than the JL in TRI because the angle between the catheter and the reverse side of the aorta is increased. Furthermore, IL in TRI is shown to be more supportive than a JL in TFI.

\subsubsection{Engagement Maneuver of Ikari Left}

The maneuver is basically the same as JL. Advance the catheter slowly into the left coronary cusp; almost always it can engage the left coronary artery without any other manipulation. There is only a small difference in the gradual angle at the reverse side of the aorta compared with JL. However, this difference enhances safety because of the motion of the engagement. Professor Ikari has trained many people how to use IL; however, for operators familiar with JL, handling the IL will come naturally.

\subsubsection{Tips to Increase Backup Force in Ikari L (Power Position)}

Occasionally, stronger backup force is necessary for an extremely complex lesion. There is an easy manipulation to increase backup force in IL. This technique is as easy as pushing the guiding catheter up to the reverse side angle of $90^{\circ}$ (black arrow; Fig. 7.7). At this point the backup force becomes much more significant because the backup force has strong relation with the angle between the guiding catheter and reverse side of the aorta. The power position with IL is safe because the distal tip is never inserted deeply due to its differentiated design since the distance is the same 

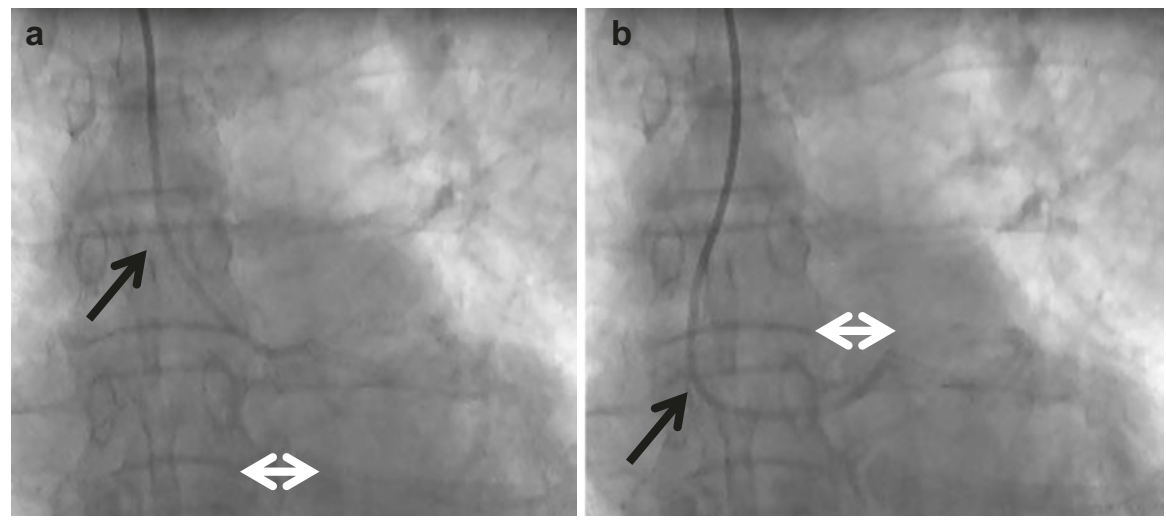

Fig. 7.7 (a) Normal position of Ikari left via right transradial approach. A black arrow shows that the angle $\theta$ is small. (b) When strong backup force is necessary, push the guiding catheter, and make the angle $90^{\circ}$ (black arrow). Note that the distal tip of the guiding catheter is not deep since the white arrows show the same distance from the vertebrate both in normal and in power positions

between normal position and power position (white arrow; Fig. 7.7). To date, no left main dissections with IL have been reported in our database (>1000 cases) and in the reports from Youssef et al. (>600 cases) [8] or other studies.

\subsubsection{Size of Ikari L}

The IL 3.5 is a regular size catheter. Most cases can be done successfully with the IL 3.5. If the aorta is elongated, IL 4.0 fits this type of aorta. The elongated aorta is found in severe atherosclerotic patients such as old hypertensive patients or patients with diffuse and complex coronary disease. In the power position, the IL 4.0 will offer even more support than the 3.5. However, we suggest IL 3.5 for your first experience.

\subsubsection{Long-tip Catheters as Voda/EBU/XB Type}

Guiding catheters can be divided into active or passive manipulation types. The EBU/XB-type guide catheters are passive type, and this may explain why they are utilized so extensively, whereas the IL or JL is an active-type catheter.

What is the benefit of the passive guide catheter? It is not necessary to continually manipulate the guide catheter during the procedure, allowing the operator to concentrate on manipulating other devices such as guidewires, balloon catheters, etc. What is the disadvantage of the passive-type guiding catheter? Common passive guide catheters are the EBU/XB type for the left coronary artery and the Amplatz L for the right coronary artery. Note that these catheters are long-tip catheters. Engagement of long-tip catheters is more difficult than a short-tip catheter. Deep insertion is inevitable with the long-tip catheters, which have higher risk for 
coronary dissection. Careful engagement is necessary. However, after safe engagement of the guide catheter, the long-tip catheter can safely advance deeper over the guidewire or during balloon catheter removal without any operator manipulation. Operators cannot perfectly control advancement of the catheter, especially during counteraction of pulling devices out from the coronary artery. That being the case, if there is significant plaque at the proximal coronary artery, there is no way to avoid coronary dissection except to avoid using long-tip catheters. The choice of catheter needs to be carefully balanced with regard to the amount of support required and the dangers of potential for pressure damping and dissection.

\subsubsection{Right Coronary Artery}

The Judkins R (JR) is a standard catheter for the right coronary artery in the transfemoral approach. Easy engagement and safety are benefits of JR. However, in TRI, the JR behaves differently because of different angles and sometimes difficult engagement. This catheter also has weak backup force when used for TRI. Thus, we need a catheter like the JR but more suitable for TRI.

\subsubsection{Ikari L for Right Coronary Artery}

The Ikari L (IL) was originally designed for the left coronary artery, but it is also good for the right coronary artery [9]. The shape of the catheter looks like the JR if a 0.035 inch guidewire is inserted (Fig. 7.8). Thus, catheter manipulation for engagement is similar to the JR. Once the guidewire is removed, the catheter will engage the right coronary artery in a very stable position. When strong backup force is necessary, you can facilitate a power position using the Ikari L catheter with marked deep engagement. Thus, the Ikari L is a strong-type Judkins $\mathrm{R}$ for TRI. In vitro experiments showed that the IL in the power position can generate a stronger backup force than JR, Amplatz L, and Ikari R (Fig. 7.9).

Note that Ikari L in the right coronary artery may naturally engage deeply in the power position. This does not happen in the left coronary artery, but you should take care for the right coronary artery. Importantly, you can still control the catheter position and engagement depth. This is markedly different from long-tip catheters.

\subsubsection{Amplatz L for Right Coronary Artery}

The Amplatz L is a passive-type catheter good for the right coronary artery. It is a long-tip catheter and can therefore be inserted deeply into the coronary artery. This can mean a higher risk for coronary dissection. The Ikari L has a similar shape to the JR (Fig. 7.10). When used in a passive way, engagement manipulation of the Ikari L is like JR, but backup force is stronger. If greater backup force is necessary for severe lesions, it is easy to make the power position by pushing the catheter along the guidewire. An in vitro study showed that backup force of the Ikari L at power position was stronger than any other catheters including Amplatz L. 
Fig. 7.8 When a 0.035

inch guidewire is inserted, the Ikari L looks like Judkins R

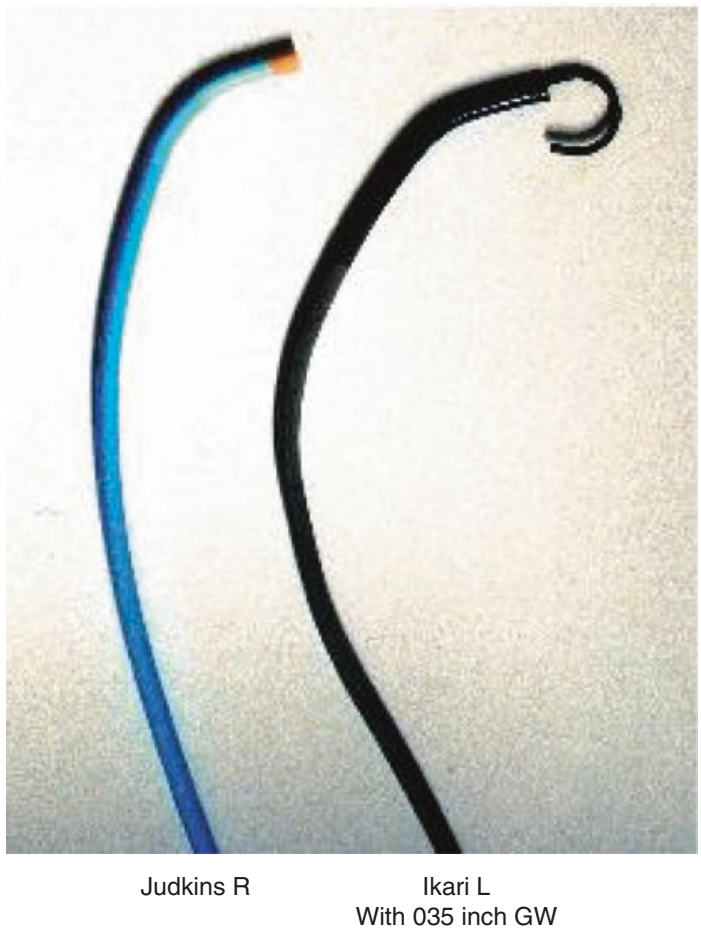

Comparison of Backup Force in TRI
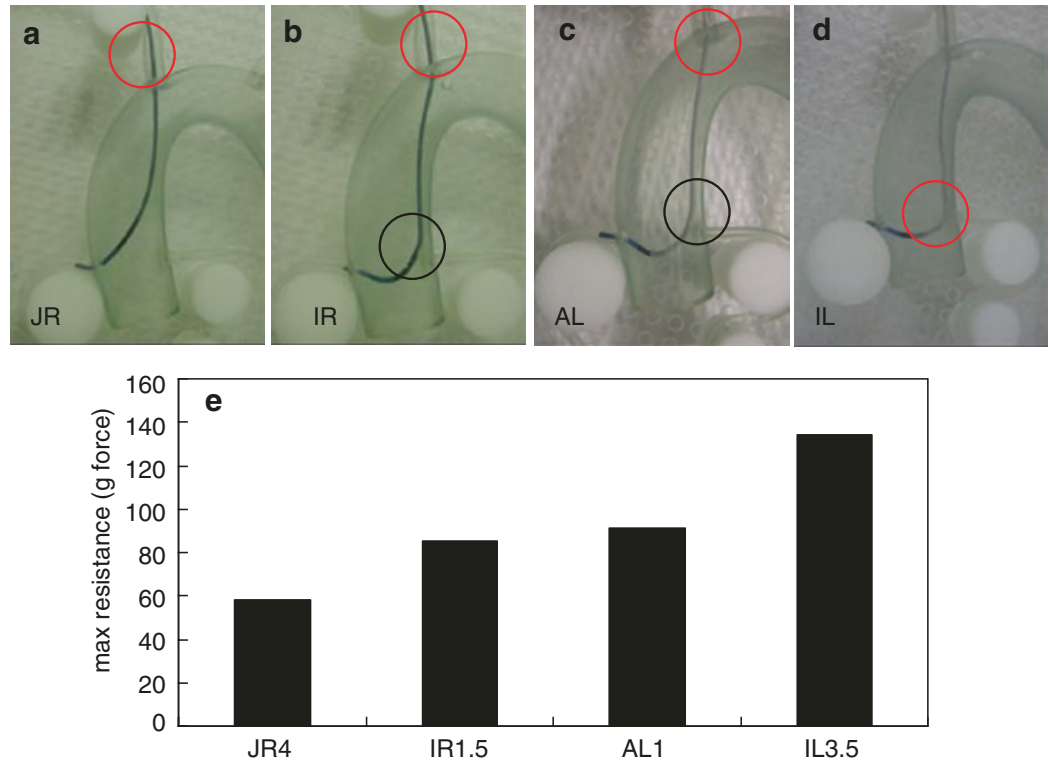

Fig. 7.9 Backup force for the right coronary artery showed Ikari L has the greatest backup force 


\section{Shape of Guiding Catheters for RCA}

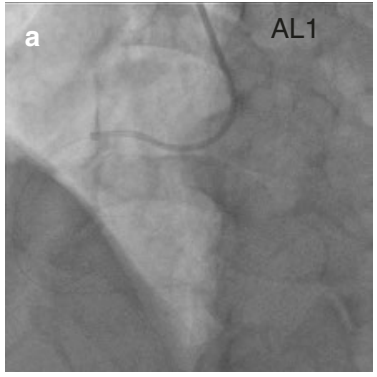

Amplatz L

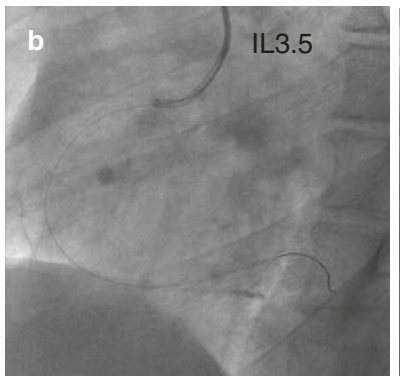

Ikari L

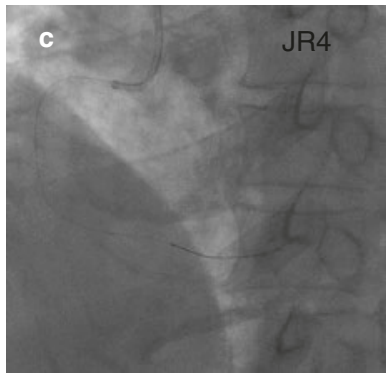

Judkins $\mathbf{R}$

Fig. 7.10 Engagement showed Ikari L looks like Judkins R

\subsection{Benefits of Universal Guiding Catheters}

Ikari $\mathrm{L}$ is a universal catheter both for the left and right coronary arteries. One great benefit is lower cost because of using a single catheter in the procedure. Secondly, a shorter procedure time in TRI may be especially important in patients with ST-segment elevation myocardial infarction. A smart way to reduce door-toballoon time is to use Ikari L [10]. Only a single catheter is necessary to perform coronary angiography both for the left and right coronary arteries and PCI for the culprit lesion which is found in either the right or left coronary artery. Torii et al. reported puncture-to-reperfusion time was significantly shorter in Ikari L use compared with conventional primary PCI (median 15 min vs. 25 min, $p=0.001$ ) [11]. Thus, Ikari L both for the left and right coronary arteries is an ideal catheter for STEMI.

\subsection{Case Report: Primary PCI Using an Ikari Guide}

Fuminobu Yoshimachi and Yuji Ikari

\subsubsection{Introduction}

Timely reperfusion is the goal in management of ST elevation myocardial infarction (STEMI). This is best achieved using primary PCI (PPCI) and ideally via a radial approach as this has been shown to reduce procedure-related complications. The Ikari left (IL) guide catheter is a useful adjunctive tool for PPCI and can provide substantial guide support in both the left and right coronary artery positions. 


\subsubsection{Case Report}

A 72-year-old male was admitted to a local hospital with chest pain due to an inferior ST-segment elevation myocardial infarction. An attempt was made to undertake PPCI to the right coronary artery (RCA) in the district hospital from a radial approach. The RCA though was a challenging vessel and due to tortuosity needed an Amplatz 1 guide catheter to gain adequate support. Unfortunately though, the operator was able to engage the RCA, the catheter had weak backup force, and although a wire would cross, a balloon would not track (Fig. 7.11a). The procedure was abandoned, and he was therefore transferred to our center for a second attempt at PPCI.
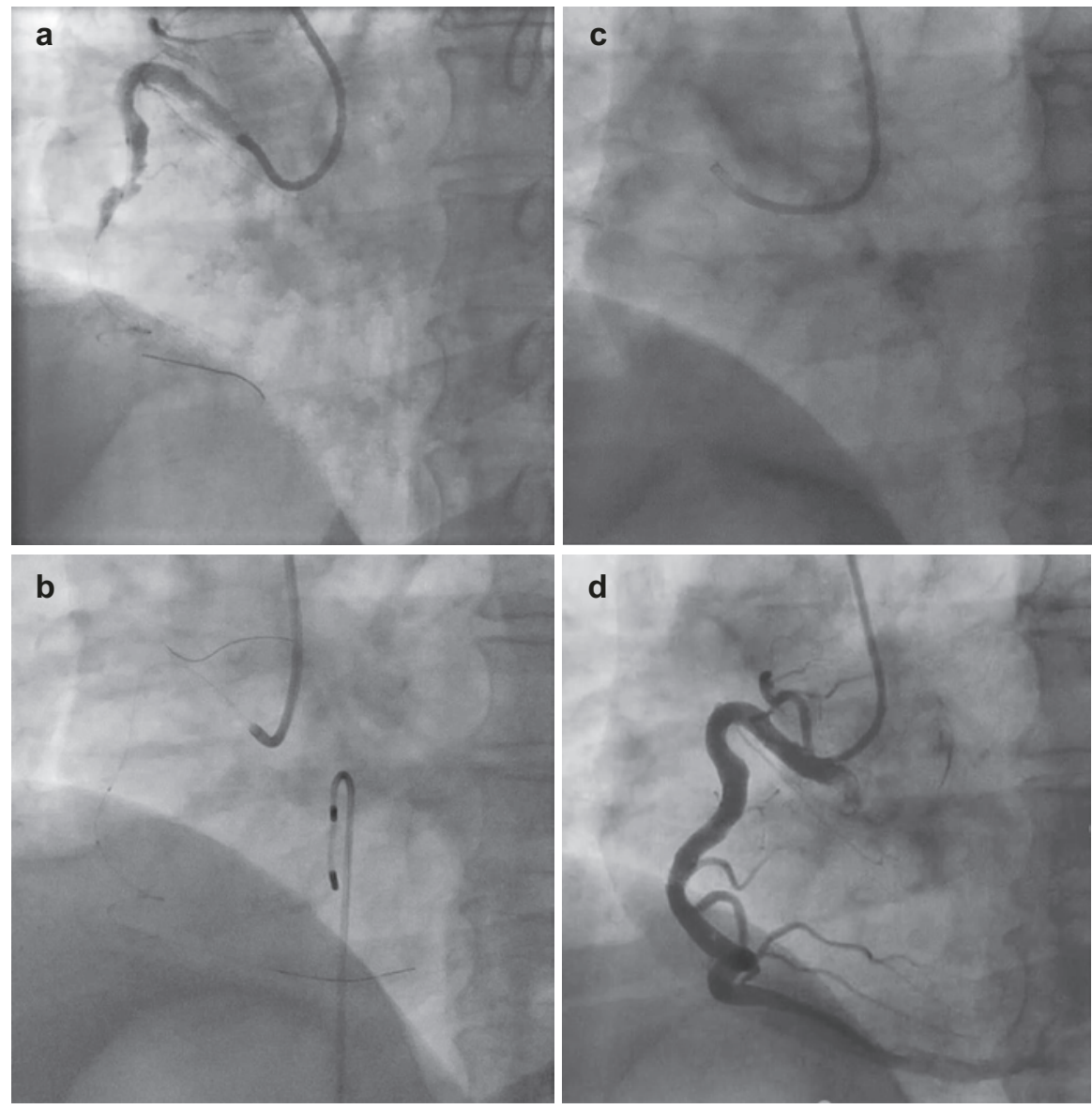

Fig. 7.11 (a) A case with inferior ST elevation myocardial infarction. Coronary angiography showed tortuous right coronary artery with total occlusion. An Amplatz left guiding catheter had insufficient backup force. A guidewire passed but no balloon passed. (b) A 7Fr guiding catheter with anchor balloon technique supported balloon pass. However, a stent could not pass the lesion due to insufficient backup force of the guiding catheter. (c) A 6Fr Ikari L 3.5 with power position engaged well into right coronary artery. (d) Final angiographic result after delivery of the stent which had passed easily due to the high backup force of the power position 
In our center, a young operator tried PPCI using 7Fr Ikari L with an anchor balloon technique (Fig. 7.11b) used to further engage the guide catheter more deeply. The balloon passed the lesion, and TIMI3 flow was achieved. The thrombus was allowed to dissolve over a few days, and he was brought back for relook angiography and stenting. This time the procedure was performed using a 6Fr Ikari L guide. In the normal position, it was not possible to pass the lesion with a stent. However, in the power position (Fig. 7.11c), a stent easily passed the lesion because of the strong backup force with excellent final result (Fig. 7.11d).

\subsubsection{Summary}

The Ikari left guide is a useful time-saving measure for patients undergoing PPCI. Moreover, in expert hands, the power position can offer greater support than possible with conventional guides, especially in the RCA.

\section{References}

1. Hofmann R, et al. Oxygen therapy in suspected acute myocardial infarction. N Engl J Med. 2017;377(13):1240-9.

2. Valgimigli M, et al. Radial versus femoral access in patients with acute coronary syndromes undergoing invasive management: a randomised multicentre trial. Lancet. 2015;385(9986):2465-76.

3. Chase AJ, et al. Association of the arterial access site at angioplasty with transfusion and mortality: the M.O.R.T.A.L study (mortality benefit of reduced transfusion after percutaneous coronary intervention via the arm or leg). Heart. 2008;94(8):1019-25. https://doi.org/10.1136/ hrt.2007.136390. Epub 2008 Mar 10

4. Seto AH, et al. Real-time ultrasound guidance facilitates transradial access. JACC Cardiovasc Interv. 2015;8(2):283-91.

5. Kiemeneij F. Left distal transradial access in the anatomical snuffbox for coronary angiography (ldTRA) and interventions (ldTRI). EuroIntervention. 2017;13:851-7.

6. Ikari Y, Nagaoka M, Kim JY, Morino Y, Tanabe T. The physics of guiding catheters for the left coronary artery in transfemoral and transradial interventions. J Invasive Cardiol. 2005;17:636-41.

7. Ikari Y, Ochiai M, Hangaishi M, Ohno M, Taguchi J, Hara K, Isshiki T, Tamura T, Yamaguchi T. Novel guide catheter for left coronary intervention via a right upper limb approach. Catheter Cardiovasc Diagn. 1998;44:244-7.

8. Youssef AA, Hsieh YK, Cheng CI, Wu CJ. A single transradial guiding catheter for right and left coronary angiography and intervention. EuroIntervention. 2008;3:475-81.

9. Ikari Y, Masuda N, Matsukage T, Ogata N, Nakazawa G, Tanabe T, Morino Y. Backup force of guiding catheters for the right coronary artery in transfemoral and transradial interventions. $\mathrm{J}$ Invasive Cardiol. 2009;21:570-4.

10. Chow J, Tan CH, Tin AS, Ong SH, Tan VH, Goh YS, Gan HW, Tan KS, Lingamanaicker J. Feasibility of transradial coronary angiography and intervention using a single Ikari left guiding catheter for ST elevation myocardial infarction. J Interv Cardiol. 2012;25:235-44.

11. Torii S, Fujii T, Murakami T, Nakazawa G, Ijichi T, Nakano M, Ohno Y, Shinozaki N, Yoshimachi F, Ikari Y. Impact of a single universal guiding catheter on door-to-balloon time in primary transradial coronary intervention for ST segment elevation myocardial infarction. Cardiovasc Interv Ther. 2017;32:114-9. 
Open Access This chapter is licensed under the terms of the Creative Commons Attribution 4.0 International License (http://creativecommons.org/licenses/by/4.0/), which permits use, sharing, adaptation, distribution and reproduction in any medium or format, as long as you give appropriate credit to the original author(s) and the source, provide a link to the Creative Commons license and indicate if changes were made.

The images or other third party material in this chapter are included in the chapter's Creative Commons license, unless indicated otherwise in a credit line to the material. If material is not included in the chapter's Creative Commons license and your intended use is not permitted by statutory regulation or exceeds the permitted use, you will need to obtain permission directly from the copyright holder. 